\title{
Avoiding Barriers to PET Radioligand Development: Cellular Assays of Brain Efflux Transporters
}

$\mathbf{P}$ and powerful means for studying a clinically relevant protein target within the brain, provided that an adequately specific and sensitive radiotracer is available. Interesting targets, which include neurotransmitter receptors, neurotransmitter transporters, enzymes, and plaques, far exceed the availability of effective radiotracers. Most PET radiotracers are designed to bind avidly and reversibly with their target. This type of radiotracer is usually termed a radioligand. Candidate radioligands for imaging must ideally satisfy an array of demanding criteria. These include ability to penetrate the blood-

\section{See page 415}

brain barrier (BBB), high affinity relative to target density (i.e., a high ratio of receptor concentration $\left(B_{\max }\right.$, $\mathrm{nM})$ to equilibrium dissociation constant $\left(K_{\mathrm{d}}, \mathrm{nM}\right)$, selectivity for binding to the target versus off-target sites (i.e., relatively low $B_{\max } / K_{\mathrm{d}}$ for off-target sites), low nonspecific binding, lack of troublesome radiometabolites in the brain, and amenability to labeling with a positron emitter, such as ${ }^{11} \mathrm{C}$ (half-life, $20.4 \mathrm{~min}$ ) or ${ }^{18} \mathrm{~F}$ (half-life, $109.7 \mathrm{~min}$ ) (1-3). Among these criteria, as for central nervous system drugs, the ability of a candidate radioligand to cross the $\mathrm{BBB}$ is of course crucial but is also notoriously difficult to predict with any certainty.

PET radioligands are typically small druglike molecules, and thus passive

Received Sep. 7, 2010; revision accepted Sep. 15, 2010.

COPYRIGHT (C) 2011 by the Society of Nuclear Medicine, Inc.

DOI: 10.2967/jnumed.110.081125 diffusion across the lipid bilayer of the BBB is usually the sole mechanism available for their entry from blood into brain. Passive diffusion is favored for compounds below $500 \mathrm{Da}$ in molecular weight and having a moderate lipophilicity represented by a $\log \mathrm{D}$ value in the range 1.5-3.0 (optimally 2). Exceptionally, some PET radioligands with either low or high $\log \mathrm{D}$ values are also known to enter the brain adequately $(4,5)$. Other molecular properties that favor brain entry by passive diffusion are absence of a formal charge in a major species of the radioligand, moderate polar surface area, and low numbers of hydrogen bond donor or acceptor groups (6). Most successful PET radioligands have these properties. Of special concern are candidate radioligands that appear to satisfy the recognized criteria for brain entry by passive diffusion, on the basis of their molecular properties, but that are found experimentally in human PET experiments to show very low or virtually insignificant brain entry. Examples of candidates showing such behavior are in fact quite common $(7,8)$. It is increasingly recognized that many such candidates fail because they are likely intercepted by one or more efflux transporters at the BBB.

Efflux transporters belong to the adenosine triphosphate-binding cassette (ABC) superfamily. They are widely expressed throughout the body and have a protective and excretory role, acting to prevent the transit of xenobiotics across organ membranes. At the BBB, efflux transporters act to prevent the influx of potentially toxic compounds. Their over- or underexpression is also postulated to contribute to neuropsychiatric and neurodegenerative disorders and to drug resistance in some diseases, such as epilepsy (9). The most active efflux transporters at the $\mathrm{BBB}$ are $\mathrm{P}$ glycoprotein (P-gp: ABCB1, multidrug resistance [MDR1]), breast cancer resistance protein (BCRP: ABCG2), and the MDR-associated protein (ABCC) family that transports anionic and glutathione-conjugated drugs (10). From the standpoint of developing successful PET radioligands that are able to cross the human BBB by escaping the action of efflux transporters, two severe issues arise. The first is that substrate behavior for efflux transporters such as $\mathrm{P}-\mathrm{gp}$ is not at all accurately predictable from molecular structure (11). Although certain properties such as high lipophilicity and high molecular weight generally promote substrate behavior, even quite small changes in structure may affect substrate avidity. Thus, for example, very structurally related $N$-alkyl-2-methoxyphenylpiperidines, which have featured strongly as PET radioligands (e.g., ${ }^{18} \mathrm{~F}-\mathrm{MPPF},{ }^{11} \mathrm{C}-\mathrm{WAY}$, ${ }^{11} \mathrm{C}$-RWAY, and ${ }^{11} \mathrm{C}-\mathrm{CUMI}-101$ [[Omethyl- $\left.{ }^{11} \mathrm{C}\right] 2-(4-(4-(2-m e t h o x y p h e n y l)$ piperazin-1-yl)butyl)-4-methyl-1,2,4triazine-3,5(2H,4H)dione]) for imaging brain 5-hydroxytryptamine receptor 1A, show a range of efflux transporter avidity in rodents (12-15). Hence, it is relatively difficult to avoid or remove transporter substrate behavior by rational molecular design without detrimentally impinging on the other desired radioligand qualities. The second issue is that substrate behavior can be strongly species-dependent (16), as may be exemplified with ${ }^{11} \mathrm{C}$-RWAY, which is an avid P-gp substrate in rodents (14) but not in monkeys (17).

The transfer of a candidate PET radioligand for brain imaging to evaluation in healthy human subjects based on findings in other species, typically rodent or monkey, requires considerable resources, time, and effort, especially to 
show adequate safety. Therefore, the availability of an in vitro screen that might reliably predict small-molecule substrate avidity for human BBB efflux transporters would be a valuable tool for streamlining PET radioligand development. The article in this issue of The Journal of Nuclear Medicine by Tournier et al. (18) attempts to address this need. The authors used MadinDarby canine kidney II (MDCKII) cells transfected with either human $M D R I$ or $B C R P$ to perform bidirectional transport assays to assess the transport susceptibility of compounds that are regularly labeled for brain PET studies. Many of the tested compounds are PET radiotracers in human use and were revealed not to be substrates of either transporter. These included $p$-MPPF, WAY-100635, and flumazenil, which had previously appeared to be P-gp substrates in rodents $(8,12)$. The main exceptions were the known P-gp substrates, prazosin, verapamil, and loperamide. Hence, this type of assay holds promise as a valuable first-pass screen for human P-gp or BCRP substrate behavior for prospective PET radioligands before their demanding preparation and evaluation with PET in animals and especially before their evaluation in human subjects. This is a clear step forward from current reliance on experiments in animals such as in transporter knockout mice $(15-19)$ or in animals for which transporters have been blocked selectively with inhibitors $(8,12,16,17)$.

Nevertheless, there is some need for caution with regard to these new assays, and there are aspects that might be improved with further development. Although the transfected transporters are human, the cell line background is canine. As a polarized epithelial kidney cell line, the MDCKII cells have a background level of canine transporters, with uncharacterized expression and species differences in substrate recognition. Such a possibility may explain one unexpected finding by Tournier et al. (18), that loperamide and verapamil were transported in the reverse direction in the absence of functional P-gp. Incorporation of control untransfected MDCKII cells might partly resolve these problems. Cells transfected with a comprehensive panel of transporters, including MDR-associated protein transporters, would be required to ensure that all transportable chemical space is covered. At the most simplistic level, the important overall question is whether energy-dependent basal-to-apical transport will be seen for a candidate PET radioligand in humans in vivo. Eventually, the answer to this question might be better provided by a model using an immortalized human brain endothelial cell line. Transport could be confirmed with either metabolic inhibitors (indicating energy dependence) or specific inhibitors of each transporter. This model would remove concerns arising from background transporter levels in addition to species variations. Finally, PET in combination with transporter substrate radiotracers, such as ${ }^{11} \mathrm{C}$-verapamil (20) and ${ }^{11} \mathrm{C}-\mathrm{N}$-desmethyl-loperamide (21), has increasing potential for elucidating the role of BBB efflux transporters in pathophysiology (22). In vitro assays that reliably quantify human transporter substrate behavior may ultimately be helpful for developing a new generation of selective and sensitive radiotracers of transporter function.

Perhaps the key message from the paper of Tournier et al. (18) is that species differences in transporter function exist and that animal models for PET studies should be used with caution. For PET radioligand development, an understanding of candidate interactions with transporters at the BBB is crucial and should be gained earlier rather than later in the development pathway. Cell biologic assays, such as those described by Tournier et al. (18), are a step forward in bridging this knowledge gap.

\section{ACKNOWLEDGMENT}

Our work is supported by the Intramural Research Program of the National Institutes of Health (NIH, NCI, and NIMH).
Matthew D. Hall

Victor W. Pike

National Institutes of Health

Bethesda, Maryland

\section{REFERENCES}

1. Pike VW. Positron-emitting radioligands for studies in vivo: probes for human psychopharmacology. J Psychopharmacol. 1993;7:139-158.

2. Laruelle M, Slifstein M, Huang Y. Relationships between radiotracer properties and image quality in molecular imaging of the brain with positron emission tomography. Mol Imaging Biol. 2003;5: 363-375.

3. Patel S, Gibson R. In vivo site-directed radiotracers: a mini-review. Nucl Med Biol. 2008;35: 805-815.

4. Waterhouse RN. Determination of lipophilicity and its use as a predictor of blood-brain barrier penetration of molecular imaging agents. $\mathrm{Mol}$ Imaging Biol. 2003;5:376-389.

5. Pike VW. PET Radiotracers: crossing the bloodbrain barrier and surviving metabolism. Trends Pharmacol Sci. 2009;30:431-440.

6. Hitchcock SA. Blood-brain barrier permeability considerations for CNS-targeted compound library design. Curr Opin Chem Biol. 2008;12: 318-323.

7. Elsinga PH, Hendrikse NH, Bart J, Vaalburg W, van Waarde A. PET studies on P-glycoprotein function in the blood-brain barrier: how it affects uptake and binding of drugs within the CNS. Curr Pharm Des. 2004;10:1493-1503.

8. Ishiwata K, Kawamura K, Yanai K, Hendrikse NH. In vivo evaluation of P-glycoprotein modulation of 8 PET radioligands used clinically. J Nucl Med. 2007;48:81-87.

9. Palmer AM. The role of the blood-CNS barrier in CNS disorders and their treatment. Neurobiol Dis. 2010;37:3-12.

10. Loscher W, Potschka H. Blood-brain barrier active efflux transporters: ATP-binding cassette gene family. NeuroRx. 2005;2:86-98.

11. Raub TJ. P-glycoprotein recognition of substrates and circumvention through rational drug design. Mol Pharmacol. 2006;3:3-25.

12. Elsinga $\mathrm{PH}$, Hendrikse $\mathrm{NH}$, Bart $\mathrm{J}$, van Waarde A, Vaalburg W. Positron emission tomography studies on binding of central nervous system drugs and P-glycoprotein function in the rodent brain. Mol Imaging Biol. 2005;7: 37-44.

13. Passchier J, van Waarde A, Doze P, Elsinga PH, Vaalburg W. Influence of P-glycoprotein on brain uptake of $\left[{ }^{18} \mathrm{~F}\right] \mathrm{MPPF}$ in rats. Eur $J$ Pharmacol. 2000;407:273-280.

14. Liow JS, Lu S, McCarron JA, et al. Effect of a Pglycoprotein inhibitor, cyclosporine A, on the disposition in rodent brain and blood of the 5-HT1A receptor radioligand, $\left[{ }^{11} \mathrm{C}\right](-)$ RWAY. Synapse. 2007;61:96-105.

15. Liow JS, Lu S, Zoghbi SS, et al. $\left[{ }^{11} \mathrm{C}\right] \mathrm{CUMI}-101$, an agonist radioligand for serotonin 5-HT1A receptors, also binds to brain alpha1-adrenoceptors in rodents and monkeys. Neuroimage. 2010;52 (suppl 1):S56-S57.

16. Syvänen $S$, Lindhe $O$, Palner $M$, et al. Species differences in blood-brain barrier transport of three positron emission tomography radioligands with emphasis on P-glycoprotein transport. Drug Metab Dispos. 2009;37:635-643. 
17. Yasuno F, Brown AK, McCarron JA, et al. Quantification of serotonin 5-HT1A receptors in monkey brain with $\left[{ }^{11} \mathrm{C}\right](-)$ RWAY. Synapse. 2006;60:510 520.

18. Tournier N, Valette H, Peyronneau M-A, et al. Transport of selected PET radiotracers by human P-glycoprotein $(\mathrm{ABCB} 1)$ and breast cancer resistance protein (ABCG2): an in vitro screening. $J$ Nucl Med. 2011; 52:415-423.
19. Zoghbi SS, Liow J-S, Yasuno $\mathrm{F}$, et al. ${ }^{11} \mathrm{C}$ loperamide and its $\mathrm{N}$-desmethyl radiometabolite are avid substrates for brain P-glycoprotein efflux. J Nucl Med. 2008;49:649-656.

20. Ikoma Y, Takano A, Ito H, et al. Quantitative analysis of $\left[{ }^{11} \mathrm{C}\right]$ verapamil transfer at the human blood-brain barrier for evaluation of P-glycoprotein function. J Nucl Med. 2006;47:15311537.
21. Kreisl WC, Liow JS, Kimura N, et al. P-glycoprotein at the blood-brain barrier in humans can be quantified with the substrate radiotracer $\left[{ }^{11} \mathrm{C}\right]$ N-desmethyl-loperamide. J Nucl Med. 2010;51: 559-566.

22. Kannan P, John C, Zoghbi SS, et al. Imaging the function of P-glycoprotein with radiotracers: pharmacokinetics and in vivo applications. Clin Pharmacol Ther. 2009;86:368-377. 